\title{
Identidad vocacional en el tránsito del colegio a la universidad y en los primeros años de vida universitaria
}

Lupe Jara

Universidad de Lima

Recibido: 14 de julio del 2010 / Aprobado: 19 de agosto del 2010

A partir de una muestra de adolescentes preuniversitarios $(n=59)$ se analiza el proceso de formación de su identidad vocacional durante el periodo de tránsito del colegio a la universidad. Dos años y medio después, se contacta a 38 de estos jóvenes para indagar por la evolución de esta identidad. Para la medición de esta variable se adapta la Entrevista para evaluar los Estadios de la Identidad Vocacional (EIVO), que muestra ser válida y confiable. Los resultados se discuten en función de los factores psicosociales que favorecen y dificultan la exploración y el compromiso con una opción vocacional, analizándose además la evolución del proceso de maduración de la identidad vocacional.

adolescencia / identidad vocacional / exploración / compromiso

Vocational identity from the passage of school to university and the first years of university study

The present study analizes the process of formation of vocational identity in the time period ranging from high school to the first years of university study. The initial sample consisted of 59 pre-university students, 38 of which were contacted two and a half years later to further explore the evolution of their vocational identity. Measures of the identity variable were computed using an adaptation of the Interview for the Evaluation of the Vocational Identity Stages, which shows validity and confiability. Results are discussed in function of psychosocial factors that either favor or obstruct the exploration and commitment to a vocational option. Also, the evolution of the process of maduration of the vocational identity is analized.

adolescence / vocational identity /exploration / commitment

Correo electrónico: ljara@ulima.edu.pe 
Al egresar del colegio el adolescente enfrenta un periodo de grandes cambios y de toma de decisiones importantes, condensadas en la elección del rol ocupacional que desempeñará en el mundo adulto: el joven tiene la tarea de elaborar un proyecto de vida coherente consigo mismo y con sus posibilidades, lo que implica definir no solo qué hacer en el futuro, sino fundamentalmente quién ser y, al mismo tiempo, quién no ser (Bohoslavsky, 1984).

La definición ocupacional resulta central para el adolescente, ya que será su ocupación la que determinará el uso de su tiempo y sus ingresos; de estos últimos dependerá dónde y cómo vivirá, e influirán en las redes de interacción social que establezca, lo cual, a su vez, influirá en las reacciones de los demás hacia él, y estas reacciones en las percepciones que se forme sobre sí mismo (Biehler \& Showman, 1992). Por tanto, el adolescente puede estar eligiendo su propio futuro a través de la ocupación que piensa desempeñar.

Para los adolescentes que pueden continuar con sus estudios, el acceso a roles ocupacionales pasa por la definición vocacional. Esta definición refleja una posición existencial frente al mundo desde la profesión que se elige. No se trata solo del nombre de una carrera, sino de la implementación del concepto que se tiene de sí mismo y de la búsqueda de algo que tiene que ver con la realización personal (Bohoslavsky, 1984; Osipow, 1991).
Por ello, del modo como el joven enfrente y resuelva esta tarea dependerá gran parte de su ajuste posterior y la satisfacción del rol que juegue en la sociedad. Asimismo, al ser la vocación un compromiso máximo en la vida, esta definición puede resultar amenazante para muchos adolescentes, mientras que para otros puede ser la oportunidad para sintetizar tanto su pasado como su presente, sus habilidades y limitaciones, y sus intereses y metas en la definición de una identidad coherente y estable (Biehler \& Showman, 1992; Erikson, 1993; Marcia, 1980).

Si bien este proceso se desarrolla a lo largo del ciclo vital, es en la adolescencia donde la coincidencia de diferentes factores (desarrollo de habilidades cognoscitivas, el pasaje de un ciclo educativo a otro, expectativas sociales) conduce al joven a plantearse la tarea de la definición vocacional. Este proceso pasa primero por la búsqueda y verificación de la información sobre sí mismo y sobre el mundo de los estudios y el trabajo; y luego, por la organización y reflexión de esta información para tomar una decisión acorde con las metas o proyectos personales (Casullo et al., 1996).

Así, definir este rol implica percibir las demandas y las oportunidades que la sociedad plantea e integrarlas con el bagaje individual, trazando un camino propio. Si se elige una carrera de la que apenas se conoce el nombre, la elección tendrá poco sustento y ello se re- 
flejará en mayores dificultades en el futuro (Trahtemberg, 1996). De ahí la importancia de la exploración antes de comprometerse con alguna elección.

Sin embargo, esta actividad exploratoria requiere que la persona logre tolerar la ambigüedad, incertidumbre, tensión y frustración propias de un periodo de creciente toma de decisiones (Super, en Osipow, 1991). Por ello, alcanzar una identidad vocacional madura no es fácil.

Algunos jóvenes no pueden renunciar a los elementos del pasado sobre los que sienten seguridad (Marcia, 1980) y establecen compromisos prematuros, respondiendo a las expectativas paternas, por ejemplo. Para ellos, una buena defensa es no intentar nada nuevo y buscar refugio en lo conocido (Horrocks, 1996).

En el otro lado se encuentran quienes postergan su decisión en tanto no completen su búsqueda de información, permaneciendo en un estado de moratoria (Erikson, 1993) que puede tener un impacto positivo si finalmente logran tomar una decisión coherente con ellos mismos. Aunque esta búsqueda también puede reflejar un modo de evitar el compromiso con alguna elección (Casullo et al., 1996).

Uno de los motivos para evitar la elección es la posibilidad de imaginar muchas opciones. Dado que decidirse por algo implica dejar de lado todo lo demás, aparece el conflicto, por lo que el joven toma activamente la decisión de no optar por nada. Así ninguna opción que pueda resultar gratificante es realmente abandonada, todas las cosas son posibles y deben ser mantenidas de esa forma.

Otra razón para evitar el compromiso es que el número de elecciones posibles, los intereses y actividades disponibles, puede resultar abrumador para el adolescente, quien al no saber qué camino tomar evita afrontar esta tarea, mostrándose apático o desinteresado (Biehler \& Showman, 1992; Bohoslavsky, 1984; Maurial, 1991).

\section{LOS CAMINOS POSIBLES EN EL DESARROLLO DE LA IDENTIDAD VOCACIONAL}

Marcia (1966, 1980) señala que las posibilidades de realizar una elección adecuada se relacionan con la exploración de las opciones disponibles (investigación de opciones) y el compromiso que se establezca con la opción elegida (certeza en la toma de decisiones). Dependiendo del proceso que siga, el adolescente puede ubicarse en una identidad cerrada cuando elige algo sin explorarlo, cerrándose a otras opciones (exploración mínima y compromiso firme), o puede permanecer en una identidad difusa cuando elige no elegir, manteniéndose en un estado difuso frente a un problema que en el fondo le preocupa mucho (exploración y compromiso mínimos). También puede mantenerse 
en una identidad en moratoria si demora su elección con el fin de explorar bien sus opciones (exploración fuerte y compromiso mínimo), o puede alcanzar una identidad lograda si consigue integrar en su decisión las posibilidades que ha encontrado (exploración y compromiso fuertes).

Estos caminos seguidos por los adolescentes reflejan un determinado nivel de desarrollo en el establecimiento de su identidad vocacional, lo cual no es algo invariante, pues si la exploración y el compromiso cambian, el nivel en el que se ubican también cambia. Así, la vocación puede consolidarse con el tiempo o variar hacia otras elecciones legítimas (Meeus, 1996; Waterman, 1993).

De otro lado, la ubicación en los diferentes niveles de la identidad vocacional se asocia con características particulares. Así, los de identidad lograda muestran un estilo racional, autónomo y flexible, apertura general hacia las ideas y experiencias, tendencia reflexiva, planificación lógica y mayor eficiencia en el procesamiento de la información; todo lo cual favorece que tengan una formación adaptativa de la identidad. En tanto, los de identidad cerrada, presentan un estilo normativo, convencional, pasivo y rígido; mayor conformidad con las indicaciones y expectativas de sus padres, maestros o pares; establecen compromisos sin haber reflexionado lo suficiente, negando la información que pueda contrariarlos. Dado que hacen lo que se espera de ellos, se sienten seguros y confiados, presentando bajos niveles de ansiedad. Por su parte, los de identidad en moratoria emplean un estilo flexible e intuitivo; algunos pueden encontrarse en un periodo de crisis marcado por la ansiedad y la confusión; mientras otros toman este tiempo como una oportunidad para afianzar su autonomía y examinar sus proyectos. Finalmente, los de identidad difusa reflejan un estilo evitativo, más intuitivo, impulsivo y desorganizado, caracterizado por la dilatación y demora para enfrentar directamente las tareas que les conciernen; no tienen suficiente control sobre su futuro, por lo que les resulta difícil planificarlo y tomar decisiones al respecto; pueden guiarse de las soluciones de otros, pero sus proyectos son poco consistentes; pueden o no haber explorado opciones y experimentado momentos de crisis, pero no eligen (Berzonsky, 1992; Blustein \& Phillips, 1990; Flum, 1994; Marcia, 1980; Meeus, 1992).

Este menor nivel de desarrollo de la identidad caracterizado por la ausencia de exploración parece relacionarse con la presencia de padres que dejan solos a sus hijos en esta tarea. Como afirma Trahtemberg (1996), una actitud de "haz lo que quieras, elige tú mismo", cuando el espacio de elección es muy amplio, puede ser entendida como indiferencia y falta de compromiso por parte del padre. En estos casos, si el adolescente tiene que elegir, lo más probable 
es que opte por lo más fácil o lo más cómodo, lo que le satisfaga más en el corto plazo o lo que le atemorice menos. En tanto, cuando el soporte parental existe, motiva el desarrollo de la autonomía y el proceso de individuación en la evolución del adolescente (Flum, 1994).

De otro lado, en cuanto al género, Bosma (1992), Marcia (1980) y Meeus (1992) encuentran que mientras los hombres se muestran más involucrados en su identidad vocacional, las mujeres lo están más en el área interpersonal. Asimismo, Osipow (1991) sostiene que los hombres formulan planes más realistas que les permitan ejercer un razonable control sobre su futuro, en tanto las mujeres tienden a elegir carreras más tentativas y poco orientadas. A la vez, ellas parecen más influidas por sus padres y desalentadas por la sociedad en la exploración de sus identidades vocacionales. Así, para las mujeres el mayor desarrollo de su identidad lo establece el énfasis en el compromiso (ubicándose en lograda o cerrada), mientras que para los hombres lo establece el énfasis en la exploración (ubicándose en lograda o moratoria). Sin embargo, Patterson et al. (1992) reportan que las mujeres que siguen un estilo de vida no tradicional y buscan su autorrealización a través de la carre$\mathrm{ra}$, se involucran seriamente en la adolescencia tardía en la resolución de su identidad vocacional.

\section{LA IDENTIDAD VOCACIONAL Y LA CONSTRUCCIÓN DE UN PROYECTO DE VIDA EN UN PERIODO DE TRÁNSITO}

La construcción del proyecto de vida forma parte de un proceso de maduración no solo intelectual sino también afectiva. Supone un aprender a crecer, actuar con responsabilidad y hacerse cargo de las consecuencias de las propias decisiones (Casullo et al., 1996). En síntesis, se trata de la habilidad de adquirir compromisos. A la vez, este es un momento de tránsito evolutivo, donde el egreso de la secundaria y el ingreso a la universidad representan simbólicamente el término y el pasaje a una nueva etapa, con un tránsito de roles, espacios y estatus (Halleh, 1995; McLean \& Flanigan, 1993). Estos "símbolos" le dan al adolescente la oportunidad de probar a la sociedad que es capaz de asumir un rol más adulto (Heymans, 1994). De hecho, aunque este egreso e ingreso no maduran [per-se] al individuo, sí son circunstancias que internamente les conducen a "dar el paso", utilizando estos elementos externos como un "rito de pasaje" (Carvajal, 1993).

Cuando el tránsito es exitoso, se establecen ciertos roles y valores esenciales y los jóvenes se sienten obligados y comprometidos con ellos (Stier \& Clark, 1991). Sin embargo, la complejización cada vez mayor de nuestras culturas lleva a que el adolescente requiera de períodos más largos de pre- 
paración a fin de construir un proyecto de vida maduro y coherente (Carvajal, 1993). En tal sentido, la transición no es completa, los adolescentes pueden embarcarse en una educación superior sin estar lo suficientemente equipados con todos los componentes de una personalidad adulta estable (razonamiento moral, fe en el mundo, identidad cohesiva), por lo que muchos temen hacer una elección que afecte el resto de sus vidas (Halleh, 1995).

En nuestro país, los adolescentes suelen hacer su elección vocacional sobre la base de infinitas proyecciones, muchas veces divorciadas de la realidad. Así, esperan que al egresar de la universidad, automáticamente obtendrán éxito social y económico. Y, pese a que estas expectativas pueden verse confrontadas por la realidad, nada parece suficiente como para amortiguar la fantasía paradisíaca que se deposita sobre el ingreso a ese otro mundo que simboliza la universidad (Jiménez \& Maguiña, 1992).

A este proceso se suma el cambio que implica el salir de un espacio relativamente seguro como el colegio, a un medio con demandas completamente nuevas como es la universidad. Así, se deja la rutina altamente estructurada del colegio (ambiente seguro y previsible) para pasar a un medio incierto: la universidad, donde dejan de frecuentar a su grupo de pares para asistir a clases con muchos "extraños"; cambian sus roles de estudiantes (algunos dejan de figurar como estudiantes destacados para convertirse en uno más); rinden exámenes constantes con un método de evaluación masivo e impersonal, etcétera; en su hogar las obligaciones también cambian, lo mismo que el uso de su tiempo (Jiménez \& Maguiña, 1992). A ello se agregan las marcadas brechas que existen en nuestro país entre el nivel de enseñanza de la secundaria y las universidades. De ahí que el egreso del colegio implique un momento de ajuste.

En función de lo expuesto surgen las siguientes preguntas: ¿cómo establecen los adolescentes su identidad vocacional?, ¿qué identidad vocacional alcanzan en este periodo preuniversitario?, y ¿cómo evoluciona la identidad vocacional en el tiempo? Para responder estas preguntas el estudio se plantea los siguientes objetivos:

a) Adecuar y validar la Entrevista para evaluar los Estadios de la Identidad Vocacional (EIVO).

b) Identificar y describir las características de la exploración y el compromiso realizados por los adolescentes para el establecimiento de su identidad vocacional.

c) Explorar la interacción de la exploración, el compromiso y la identidad vocacional con las variables académicas y sociodemográficas.

d) Describir la evolución de la identidad vocacional luego de dos años y medio del primer estudio. 


\section{MÉTOdO}

\section{Participantes}

Participan de este estudio 59 alumnos de una academia preuniversitaria de Lima (44,1\% hombres y 55,9\%, mujeres), entre escolares $(37,3 \%)$ y egresados del colegio $(62,7 \%)$. Su rango de edades se extendió de 15 a 19 años, siendo el promedio 17 años (50,8\%). De ellos, $78 \%$ tenía planeado postular a la universidad A y $22 \%$ a la universidad B. Los alumnos que deseaban postular a la universidad A fueron diferenciados por su rendimiento académico: alto $(37 \%)$, medio $(34,8 \%)$ y bajo $(28,3 \%)$. Del grupo inicial, luego de dos años y medio, se logró contactar a 38 jóvenes $(64,4 \%)$ para el segundo estudio (60,5\% mujeres y $39,5 \%$ hombres).

\section{Medición}

- Entrevista para evaluar los Estadios de la Identidad Vocacional (EIVO): Investiga la madurez psicosocial de la identidad vocacional (Jones, Akers \& White, 1994) sobre la base de los criterios de exploración (búsqueda de información respecto a las opciones vocacionales) y compromiso (grado de certeza respecto a la decisión tomada). Esta entrevista ha sido adaptada por Jara (2003) del manual elaborado por Grotevant y Cooper (1981), estableciendo los siguientes criterios para su codificación. La exploración puede calificarse como: (1) mínima, (2) general o confirmatoria, (3) moderada o (4) fuerte. Mientras, el compromiso puede calificarse como: (1) mínimo, (2) en proceso, (3) confirmatorio o (4) fuerte. A partir de estos puntajes, el adolescente es clasificado dentro de un estadio de la identidad:

- Identidad lograda: alto en exploración (3/4) y alto en compromiso (4).

- Identidad en moratoria: alto en exploración (3/4) y bajo en compromiso (2).

- Identidad cerrada: bajo en exploración (2/1) y alto en compromiso (3).

- Identidad difusa: bajo en exploración $(1 / 2)$ y bajo en compromiso (1).

Para esta adaptación Jara (2003) obtuvo un índice de validez de contenido de 1.0 y para la validez de constructo el análisis de componentes principales dio soporte a la definición de la identidad vocacional en función de las dimensiones de la exploración (explica el 70,8\% de la varianza) y el compromiso $(29,2 \%$ de la varianza). Asimismo, existe asociación significativa (r Spearman) entre estadio y exploración $(.76 * * * *)$, estadio y compromiso $(.64 * * * *)$, y exploración y com- 
promiso $\left(.33^{*}\right) .{ }^{1} \mathrm{~A}$ la vez, se obtuvo una confiabilidad del puntuador de 0.84 para la exploración, 0.77 para el compromiso y 0.76 para el estadio de la identidad.

- Cuestionario sobre el desarrollo de la identidad vocacional: Mediante preguntas estructuradas investiga el mantenimiento en el tiempo de la elección vocacional, la exploración de otras opciones, la satisfacción con la elección hecha y la intención de mantenerse o cambiar de opción hacia el futuro (Jara, 2003).

- Ficha académica y sociodemográfi$c a$ : Se registró sexo, edad, si es escolar o egresado, año de egreso del colegio, universidad a la que postula, carrera a la que postula, número de veces que ha postulado, preparación preuniversitaria previa, puntaje general y puesto en la academia, y puntajes en el Test de Aptitudes Diferenciales de Bennett (DAT) (numérico, verbal, abstracto y global).

\section{Procedimiento}

Inicialmente se adecuaron las preguntas de la entrevista de identidad vocacional en función de los participantes y se agregaron dos interrogantes que no figuraban en el formato inicial. La primera, introductoria a la entrevista, y la

11 En el análisis de los resultados para las correlaciones se aplicó el criterio de significación estadística $(* \mathrm{p}<.05, * * \mathrm{p}<.01, * * * \mathrm{p}<.001$ y $* * * * \mathrm{p}<.0001)$ y el criterio de Cohen $(\mathrm{r}>.2)$ (Cohen, 1988). otra, orientada a la evaluación del compromiso. Al respecto, se consultó con trece jueces expertos quienes dieron un índice de acuerdo de 1.0 para la nueva pregunta sobre el compromiso, pero respecto a las preguntas originales, expresaron dificultades para identificar si ellas se referían a la exploración o al compromiso, coincidiendo en señalar que ello dependía de las respuestas que dieran los sujetos. Lo anterior planteaba una validación de los criterios de calificación de las respuestas, ya obtenida por Maurial (1993), con un índice de acuerdo de 1.0. Luego, se realizó un piloto a tres alumnos, estableciéndose un nuevo orden en la presentación de las preguntas que brindó mayor fluidez a la entrevista. Se añadió, asimismo, una nueva pregunta orientada a evaluar la exploración, que, de acuerdo al criterio de cuatro nuevos jueces, resultaba válida y adecuada.

Posteriormente, se contactó con una academia preuniversitaria con el fin de acceder a los participantes, quienes fueron seleccionados al azar del listado total de alumnos, cubriendo las cuotas establecidas por las variables de estudio: sexo, estatus (escolar, egresado del colegio), universidad a la que postulan (A o B), y rendimiento (alto, medio o bajo) de los postulantes a la universidad A. Luego, se coordinó con los tutores la derivación de los alumnos seleccionados que hubiesen aceptado participar voluntariamente en la investigación. La aplicación de la entrevista se realizó 
hacia la mitad del ciclo de preparación con el fin de encontrar a los alumnos ya enfrentados a las demandas de la academia, pero sin la presión que la proximidad del examen de admisión podía generar. La entrevista fue aplicada en todos los casos por la autora de este estudio, garantizando la confidencialidad de la información brindada y el carácter voluntario de la participación.

Tal como lo habían sugerido los jueces, se analizó la validez y confiabilidad de la entrevista a partir de los criterios de calificación ajustados a las respuestas dadas por los participantes. Así, el análisis de contenido realizado generó nuevos criterios que fueron consultados a diez jueces expertos, quienes dieron un índice de acuerdo de 1.0 para la validez de contenido, señalando además que estos criterios resultaban más específicos, comprensibles y discriminantes que los criterios originales. Adicionalmente, se solicitó a otros cuatro jueces que contrastaran ambas versiones de los criterios de calificación, eligiéndose la versión reajustada sobre la original en un $0,69 \%$. Posteriormente, se solicitó a cinco nuevos jueces que califiquen 16 entrevistas con el fin de medir la confiabilidad del instrumento, de donde se obtuvo una correlación de 0.84 para el nivel de exploración, 0.77 para el nivel de compromiso y 0.76 para el establecimiento del estadio de la identidad. Luego de estos procedimientos se realizó un análisis de componentes principales para la validez de constructo, verificando que la exploración (explica el 70,8\% de la varianza) y el compromiso $(29,2 \%$ de la varianza) son dimensiones de la identidad vocacional. Todos estos procedimientos se dirigen a cumplir con el primer objetivo de este estudio.

Finalmente, luego de dos años y medio, al considerarse que los participantes ya tendrían definidos sus planes académicos y que aquellos que habían logrado ingresar ya habían terminado o estaban por terminar Estudios Generales, y por tanto debían definir la facultad en la que estudiarían, se buscó establecer un nuevo contacto con ellos. A través del teléfono se logró ubicar a un grupo de los participantes a quienes se les administró un cuestionario sobre el desarrollo de su identidad vocacional.

\section{RESULTADOS}

\section{Identidad vocacional}

Los participantes presentan sobre todo una exploración baja (mínima: 40,7\% y general: $20,3 \%$ ) y un compromiso confirmatorio $(49,2 \%)$ o en proceso $(23,7 \%)$. Consecuentemente, los estadios donde los adolescentes se ubican en mayor porcentaje son el cerrado $(49,2 \%)$ y el difuso $(28,8 \%)$. Mientras que en moratoria se ubican el $13,6 \%$ y en lograda solo el 8,5\%. Ello se debe a que tanto moratoria como lograda requieren un nivel de exploración fuerte, que en la muestra se presenta en una proporción pequeña (exploración fuerte: $5,1 \%$, y moderada: $16,9 \%$ ). 
Se encuentra, además, que la exploración se asocia con inteligencia (aptitud verbal: $.35^{* *}$, abstracta: $.27 *$, y general: .29*). Mientras, un menor cuestionamiento de la elección vocacional (compromiso confirmatorio) está más presente en el adolescente que lleva más tiempo egresado del colegio (.30*), preparándose para postular $\left(.31^{*}\right)$, fracasando en el intento de ingresar $\left(.27^{*}\right)$ y con menor aptitud numérica $\left(.27^{*}\right)$.

Respecto al nivel académico de los adolescentes que postulan a la universidad A, la prueba del chi-cuadrado $\left(\mathrm{X}^{2}\right)$ indica diferencias significativas en el compromiso y el estadio. Así, los de rendimiento académico alto establecen preferentemente un compromiso en proceso $(52,9 \%)$ y se ubican en mayor porcentaje en identidad cerrada $(41,2 \%)$. En tanto, los de rendimiento medio son los únicos que realizan una exploración y compromiso fuertes $(25 \%)$, por lo que son los únicos que alcanzan una identidad lograda (25\%). Finalmente, los de rendimiento académico bajo plantean únicamente una exploración baja (mínima: 53,8\%, general: $30,8 \%, \quad y$ confirmatoria: $15,4 \%)$ y un compromiso confirmatorio $(69,2 \%)$ o mínimo $(30,8 \%)$, ubicándose solo en cerrada $(69,2 \%)$ y difusa (30,8\%). En cuanto a los alumnos que tenían planeado postular a la universidad B, ellos tienden a realizar una exploración mínima (46,2\%), establecen preferentemente un compromiso confirmatorio $(61,5 \%)$ o mínimo (23,1\%), y se ubican casi totalmente en cerrada $(61,5 \%)$ y difusa $(30,8 \%)$. Estas tendencias son similares a las de rendimiento bajo que postulan a la universidad A (véase el gráfico 1).

Gráfico 1

Identidad por universidad y nivel académico en universidad A

$\begin{array}{lll}\text { Exploración Compromiso } & \text { Estadio }\end{array}$

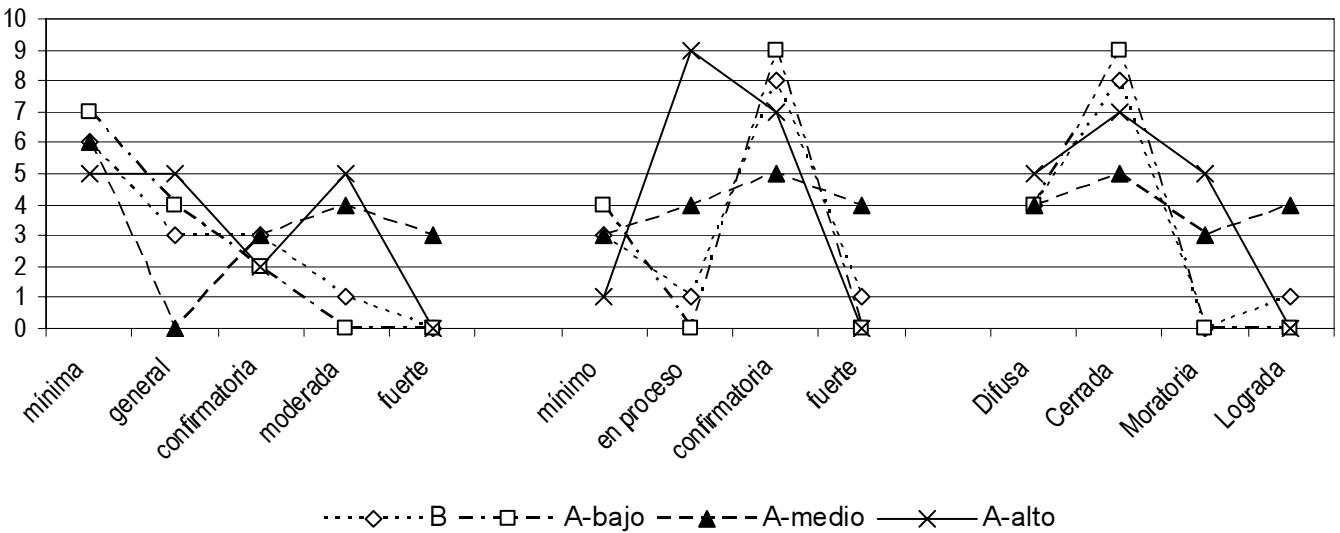


Al revisar en detalle los datos recogidos en la entrevista de identidad vocacional se encuentra que la mayoría de los adolescentes ha elegido la carrera recientemente $(52,5 \%)$, motivada particularmente por las actividades relacionadas con ella o por desarrollar sus habilidades $(52,6 \%)$, habiendo considerado previamente otras opciones $(72,9 \%)$, aunque conoce poco sobre la profesión escogida $(32,2 \%)$.

A la vez se halla que entre los adolescentes que se ubican en lograda $(\mathrm{n}=$ 5), 4 han tomado su decisión hace más de un año y todos se sienten seguros de ella, manifestando la intención de mantener esta elección. A la vez, 4 eligen la carrera por las actividades relacionadas con ella o por desarrollar sus habilidades, 4 tienen padres que les expresan satisfacción y apoyo respecto a su elección, y 3 señalan que nadie ha influido en su elección. En tanto, entre los adolescentes que se ubican en moratoria $(\mathrm{n}=8), 7$ indican que eligen la carrera por las actividades relacionadas con ella y 5 que han investigado sobre esta profesión por su cuenta. Sin embargo, 6 no se sienten seguros de esta elección y 7 dudan acerca de si la mantendrán en el futuro.

Asimismo, se encuentra que en los estadios de identidad cerrada y difusa se ubican todos los que eligen carreras administrativas, los que cuentan con poca información sobre la carrera elegida o recibieron dicha información de manera pasiva en charlas organizadas por sus colegios; los que escogen su opción motivados por diferentes modelos (padres, hermanos, profesores, entre otros) o por 'descarte'; los que tienen planes vocacionales coincidentes con los de sus padres; y los que consideran que los problemas académicos podrían interrumpir sus planes. Del mismo modo en estos estadios se ubican la mayor parte de los que señalan como principal influencia en su decisión a su familia (difusa: 33,3\% y cerrada: 61,9\%), personas cercanas, profesores y otros modelos (difusa y cerrada: $35,7 \%$ ); los que indican que les atrae el ambiente donde trabajarían (difusa y cerrada: $46,2 \%$ ) y los contactos que proporciona la carrera (difusa y cerrada: $36,4 \%$ ); los que enfocan sus planes hacia la meta inmediata: ingresar (difusa: $41,7 \%$ y cerrada: $33,3 \%$ ) y los que frente a las dificultades que podrían interferir sus metas, cambiarían de planes (difusa: 47,4\% y cerrada: 42,1\%).

A ello se agrega que tienden a ubicarse en cerrada los egresados del colegio $(59,5 \%)$, los que eligen carreras de ciencias $(66,7 \%)$ (ninguno de ciencias establece un compromiso mínimo) y los que son atraídos por la rentabilidad de la carrera $(72,7 \%)$. La mayor parte de estos adolescentes ha elegido hace más de un año la carrera $(62,1 \%)$ y se siente segura de ella $(75,9 \%)$, por lo que piensa mantener esta opción (70\%). Ellos tienden a informarse sobre su opción preguntando a familia- 
res o amigos (87,5\%), aunque sus padres los estimulan a que elijan por ellos mismos $(52,9 \%)$ y aceptan sus decisiones $(60,6 \%)$. Respecto a los de difusa, se ubican en este estadio todos los que sienten que sus padres no intervienen $(n=3)$ y la mayoría de los que consideran que sus padres están en desacuerdo con su elección (62,5\%). El $88,2 \%$ de los de difusa han elegido su opción vocacional recientemente. Los escolares $(36,4 \%)$ tienden a ubicarse en este estadio.

\section{LA IDENTIDAD VOCACIONAL A TRAVÉS DEL TIEMPO}

Entre los participantes que se logró contactar luego de dos años y medio se encontró que el ingreso a la universidad se asocia tanto a sus aptitudes diferenciales (aptitud verbal: .53***, abstracta: $.35^{*}$, numérica: $.40^{*}$ y general: $.50^{* * *}$ ) como a su rendimiento académico durante el periodo de preparación (puntaje: $.38^{*}$, puesto $.33^{*}$ y rendimiento académico: $.47^{* *}$ ); independientemente de la identidad en la que se ubiquen. Sin embargo, entre los contactados de moratoria $(n=7)$ y lograda $(n=3)$, el $70 \%$ ingresa en el primer examen y el $30 \%$ en el segundo. A la vez, la satisfacción con la carrera elegida se asocia al estadio de identidad $\left(.43^{* *}\right)$. Así, todos los de identidad lograda se encuentran "muy satisfechos" y el $85,7 \%$ de los que se ubicaron en moratoria muestra altos niveles de satisfacción.
De otro lado encontramos que luego de ingresar, la mayoría exploró otras carreras durante el periodo de Estudios Generales $(52,8 \%)$, siendo la tendencia a confirmar la opción inicial $(67,6 \%)$, así como a expresar satisfacción $(65,8 \%)$ y la intención de mantenerse en la profesión elegida (64,9\%).

Entre los jóvenes que contrastaron su opción inicial con otras carreras, la mitad todavía no se siente segura de su elección $(50 \%)$. De ahí que en este grupo se tiende a expresar insatisfacción $(55 \%)$ e inseguridad $(26,3 \%)$ o la intención de cambiar $(36,8 \%)$ de opción profesional. Prefirieron considerar otras opciones la mayor parte de los que eran escolares $(64,3 \%)$, los que ingresaron a carreras de ciencias $(77,8 \%)$, los que postulaban a la universidad A con rendimiento medio $(66,7 \%)$ y alto $(64,3 \%)$ y los que se ubicaron en difusa $(66,7 \%)$.

En tanto, entre los jóvenes que señalan no haber considerado otras opciones $(47,2 \%)$, todos indican sentirse seguros con la carrera que vienen estudiando y casi todos señalan estar satisfechos $(61,1 \%)$ o muy satisfechos $(27,8 \%)$ con esta elección, por lo que expresan la intención de mantenerse en ella $(94,4 \%)$. Prefieren no explorar otras opciones la mayor parte de los que tenían un nivel académico bajo $(66,7 \%)$, los que ingresaron a la universidad B $(75 \%)$ y a carreras de administración $(66,7 \%)$ y los que se ubicaron en cerrada $(52,9 \%)$. 
Asimismo, de manera específica encontramos que entre los que postulaban a la universidad A con rendimiento alto, la mayor parte refiere sentirse segura $(57,1 \%)$ y satisfecha con la carrera elegida $(57,1 \%)$, pero también la mayoría indica no saber si mantendrán esta decisión $(57,1 \%)$. Asimismo, más de la mitad de los que eran egresados no exploraron otras opciones $(54,5 \%)$ y se sienten insatisfechos con la carrera que vienen estudiando $(52,2 \%)$. Pese a ello, también la mayoría indica que se siente segura de su opción $(59,1 \%)$ y que se mantendrá en ella $(54,5 \%)$.

\section{DISCUSIÓN}

Encontrar la ocupación ideal con la que luego se participará en la vida adulta es una tarea compleja para muchos adolescentes. Elegir quién ser en el futuro no resulta fácil. El joven realiza esta elección en un momento de tránsito y pérdida de los referentes que estructuraban sus roles y sus vínculos; y cuando el entorno adulto centra su atención en él, consciente de la importancia de su decisión. Sin embargo, el interés se enfoca en el rendimiento más que en la vocación misma. Entonces, ¿cómo eligen los adolescentes la carrera de toda la vida?, y ¿cuánto conocen y cuán comprometidos se encuentran con esta elección?

La mayor parte de los adolescentes realiza su elección vocacional en un tiempo muy breve, guiado por las actividades propias de la carrera y por la expectativa de que esta le permita desarrollar sus habilidades. Sin embargo, no todos realizan una exploración fuerte de sus opciones. Es decir, muchos postulan a una carrera de la que conocen muy poco y si bien han contrastado diferentes opciones, esta comparación ha sido superficial. En tal sentido, no parece que hayan reflexionado lo suficiente sobre su decisión, por lo que difícilmente ella puede integrar los diferentes aspectos del joven o reflejar quién es y quién quiere llegar a ser. La tarea se dificulta además debido a que existe una variedad de posibilidades ofertadas por el medio, pero muy poco tiempo para explorarlas. La meta, por tanto, no está puesta en la elección de una carrera que refleje lo que el adolescente quiere llegar a ser, sino en el ingreso. Así, el adolescente recibe las múltiples ofertas vocacionales y elige una para enfrentar la tarea inmediata de postular, dejando de lado el análisis y el esclarecimiento de su vocación.

Frente a ello, una alternativa sería postergar el compromiso en tanto se espera un tiempo de menor presión - como podría ser el periodo de los Estudios Generales- para explorar con mayor detenimiento diferentes opciones vocacionales, estableciendo en consecuencia una identidad en moratoria. No obstante, este camino es seguido por muy pocos adolescentes (14\%), la mayoría realiza un compromiso 
firme en el que evita cuestionar su decisión. Ello, especialmente, si cuenta con menores aptitudes intelectuales y ya lleva un tiempo fracasando en el intento de ingresar. En tanto, otro grupo numeroso tiende a mostrarse poco involucrado en la tarea de explorar sus opciones, con lo cual los estadios que predominan son el cerrado y el difuso.

Las dificultades para alcanzar un estadio de identidad de mayor desarrollo (lograda o moratoria) parecen encontrarse tanto en los factores externos como en los internos. Por un lado, en el entorno se espera que al finalizar la etapa escolar el adolescente se encuentre en condiciones de elegir la carrera que desea y lo presiona hacia ello. Y, ante sus dudas normales, la presión se dirige hacia el ingreso. No se crean las condiciones necesarias para abrir un espacio donde tenga lugar el proceso reflexivo, pues se entiende como lógico y necesario el pasaje del colegio a la universidad con el fin de continuar con la formación académica. De esta mane$\mathrm{ra}$, aunque la meta puesta en el ingreso se define claramente, se deja de lado el proceso implicado en ella.

En relación con aspectos más bien internos, muy pocos cuentan con las condiciones necesarias para reflejar en su elección un adecuado conocimiento de sí mismos. De ahí que solo cinco adolescentes se ubican en lograda. Ellos venían considerando su opción con bastante anticipación, tiempo en el cual, de una u otra manera, habían tenido experiencias previas relacionadas con la carrera elegida, en las que habían contrastado sus aptitudes e intereses, recibiendo el apoyo explícito de sus padres, quienes posteriormente también hicieron notar su satisfacción con la decisión tomada por sus hijos. Estos adolescentes manifiestan que tomaron por sí mismos su decisión, sin la influencia de su familia. Así, estos jóvenes contaron con el apoyo de un entorno empáticamente involucrado, que favorecía que ellos elijan libremente, pero sin dejarlos solos.

En tanto, la mayoría ubicada en identidad cerrada (49\%), aunque se mostraba segura de su opción, no tenía un conocimiento suficiente sobre la carrera a la que postulaba ni sobre las habilidades y aptitudes requeridas por esta, pese a haber realizado su elección con anticipación. En tal sentido, su decisión respondía más a factores externos y aleatorios, como la influencia de modelos o pares, lo que ofertaba el mercado, alguna carrera de moda, el ambiente de trabajo, entre otros, que a un interés personal, con lo cual, esta elección puede ser fuente de frustración más adelante. $\mathrm{Al}$ respecto, resulta significativo que los adolescentes que postulaban a carreras de ciencias tiendan a ubicarse en este estadio, mostrando una seguridad temprana, respecto a una opción poco explorada. De otro lado, aunque la mayoría de estos jóvenes señalan que sus padres los estimulan a que elijan por 
sí mismos y aceptan la decisión que ellos toman, también indican que sus planes vocacionales coinciden con los planes que sus padres tenían para ellos, y cuando estos planes difieren, suelen reflejar la influencia de algún familiar o modelo. Vemos que, por un lado, aunque los padres expresan de manera explícita su apoyo, no dejan de manifestar sus deseos sobre sus hijos, deseos que son asumidos por estos en la elección final de la carrera. Y, por el otro lado, ante la libertad de elegir, lo más seguro resulta adherirse a una vocación conocida, especialmente si quien la detenta es un modelo de prestigio para el adolescente.

De esta manera, la expresión de un compromiso categórico, sin haber explorado lo suficiente o a priori, puede ser una manera de responder a las expectativas depositadas por el entorno -padres, pares, modelos- en el joven. A la vez que una forma de protegerse a sí mismos de vivir en medio de una situación desestructurada y novedosa, sin un sostenimiento adecuado por parte del entorno y más bien presionado por este, que pretende ofertar lo "mejor" para él, enfatizando la importancia del ingreso.

El adolescente puede preferir optar por una carrera y ser firme en esta decisión, que responder con un "no sé todavía", agregando incertidumbre al momento que vive. Desde los adultos puede existir la tendencia a censurar esta respuesta como señal de poca madurez y poco interés, cuando en realidad se trata de un momento de exploración más que de toma de decisiones firmes. También, "saber lo que se va a hacer" es encontrarse dentro del grupo de pares "adecuados" y para sí mismo, sentir que se mantiene el control y la seguridad de la situación. Por el contrario, cuestionar su elección ante la proximidad del examen de admisión lo llevaría a perder la tranquilidad que le trae tener esta certeza.

De otro lado, los adolescentes de identidad difusa (29\%), que eligen una carrera momentánea y recientemente, sin tener mayor conocimiento y convencimiento de ella, pueden estar respondiendo a la compleja tarea de formación de su identidad vocacional, evitando involucrarse en esta. Así, renuncian a asumir un compromiso bajo su propio riesgo y prefieren optar -transitoriamente- por algo decidido desde afuera, trasladando una responsabilidad que les compete a su entorno. De este modo, estos jóvenes pueden tener la sensación ilusoria de no ser ellos quienes fracasan, pues no son ellos quienes deciden. Asimismo, dado que los escolares tienden a responder de esta manera, el estadio de identidad difusa parece reflejar una posición menos madura de estos adolescentes. Algunos de ellos eligen la carrera "por descarte", evitando el riesgo de depositar en una opción todo su deseo e interés. A la vez, la mayoría señala que ante las dificultades "cambiaría de 
opción", por lo que el fracaso en una meta que es intercambiable reduce la posibilidad de frustración. Llama la atención que los adolescentes que sienten que sus padres "no intervienen" se ubican en identidad difusa, mostrando que incluso una actitud neutral de los padres puede ser vivenciada como desinterés o indiferencia, en un momento en que todavía requieren que sus elecciones sean validadas por sus autoridades.

En tanto, los de rendimiento bajo solo se ubicaron en identidad cerrada o difusa. Mientras que los de rendimiento alto se ubicaron preferentemente en cerrada y ninguno en lograda, quienes a su vez contaban con un rendimiento medio. Por otro lado, la realización de una exploración fuerte se asocia a mayores aptitudes intelectuales; mientras el establecimiento de un compromiso confirmatorio se asocia a menores aptitudes intelectuales, especialmente numéricas.

Estos datos nos llevan a plantear que la tarea de búsqueda y profundización en el conocimiento de una determinada carrera, que sintetice lo que el adolescente quiere llegar a ser, requiere de condiciones o aptitudes medianamente sofisticadas para su contraste y reflexión. Y alcanzar una identidad más desarrollada, además de recursos complejos, también requiere de interés, motivación o necesidad para cuestionarse. Así, los de rendimiento bajo, al no contar con los recursos suficientes, solo pueden ubicarse en los estadios de menor desarrollo evolutivo: cerrada y difusa. En tanto, los de rendimiento alto, al experimentar éxito en su desempeño y facilidad para resolver las tareas o demandas que el medio les plantea, pueden estar menos motivados a cuestionar sus decisiones, sus acciones o a sí mismos; sobre todo si deben enfocar sus recursos al manejo de una situación en la que se pone a prueba sus capacidades y habilidades en un examen de ingreso. Mientras, los de rendimiento medio, enfrentados a la misma tarea y contando con importantes recursos, al no ser parte de los más exitosos y al experimentar reveses pueden verse cuestionados en su desempeño, cuestionamiento que puede llevarlos a mirarse más críticamente y reflexionar sobre sus recursos y sus decisiones, los que en este momento se enfocan al ámbito vocacional. Ello, aunado a sus recursos, les permite alcanzar el estadio de identidad lograda. Otra posibilidad es que los de lograda, al estar más interesados en resolver la tarea vocacional, dediquen menos esfuerzos a su desempeño académico, confiados en que sus recursos son suficientes para alcanzar la meta del ingreso. De esta manera el nivel que muestran es menor al que potencialmente podrían actualizar. Una señal de los recursos que poseen los de lograda y moratoria es que la mayoría de ellos ingresaron en el primer examen de admisión (luego de la entrevista) y el resto en el siguiente. 
Todos los que eligen carreras administrativas (administración, economía y contabilidad) y la casi totalidad de los que postulan a la universidad B (92\%), se ubican en cerrada o difusa: ¿Qué atribuciones -explícitas o implícitasles asignan a estas carreras y a esta universidad, los jóvenes que muestran poco interés por conocer la carrera a la que postulan y poco compromiso o un compromiso no reflexivo con la decisión que han tomado?, y ¿cuál es la evaluación que hacen de sí mismos y de sus recursos?

Encontramos que los que postulan a la universidad B tienen casi el mismo perfil que los alumnos de nivel bajo que postulan a la universidad A. Lo que parece indicar que, al margen de los recursos con los que estos jóvenes cuentan, su desenvolvimiento denota poca seguridad interna, que se expresa en un esfuerzo y compromiso mínimo con una tarea vital, como es la definición vocacional.

En relación con la motivación, los de cerrada y difusa eligen carreras por el ambiente, los contactos y la rentabilidad de esta; mientras que los de lograda y moratoria realizan sus elecciones por las actividades relacionadas con la profesión y por las habilidades personales que les permite desarrollar. De esta manera, aunque la mayoría de los jóvenes se proyectan en el futuro al ámbito laboral de la profesión elegida, los de cerrada y difusa se concentran en los aspectos externos de esta, conside- rando lo que se obtiene de ella. En tanto, los de lograda y moratoria se enfocan a los aspectos intrínsecos de la carrera considerando lo que se hace en ella y lo que se es con ella; es decir, se enfocan a los aspectos vocacionales de la profesión. De ahí que sean los de cerrada y difusa los que señalen como obstáculos en la realización de sus metas, en el mediano plazo, las dificultades personales (de habilidad, capacidad o motivación) y las académicas (rendimiento); y, en el largo plazo, las dificultades laborales (competencia, desempleo, condiciones de trabajo).

En el segundo estudio, al recontactar a los jóvenes, se encuentra que luego del ingreso, durante el periodo de Estudios Generales, la mayoría de ellos explora diferentes opciones a las que tomaron inicialmente. Esta etapa, al presentar en sus cursos diferentes opciones académicas, representa una oportunidad para que los adolescentes que no han contrastado posibilidades puedan conocer con cierta profundidad y extensión aquellas alternativas más adecuadas para ellos. De esta manera, estos años de Estudios Generales se constituyen en un momento clave, donde el joven tiene la ocasión de transitar hacia una elección que realmente refleje su vocación. De ahí que la identidad evolucione; así, al incrementarse la exploración, los que se encontraban en el estadio de identidad difusa y los que ingresaron a carreras de ciencias ubicados en cerrada, transitaron hacia 
una identidad en moratoria. Es importante también notar que este camino lo siguen los más jóvenes (los que eran escolares) que no habían tenido antes el tiempo para analizar las carreras disponibles y aquellos con recursos, que parecen permitirles enfrentar la insatisfacción, inseguridad o dudas que implica el pensar sus elecciones.

Al explorar otras opciones surgen dudas respecto a si se encuentran estudiando la carrera correcta para ellos. Así, los de rendimiento alto que ingresaron a la universidad $\mathrm{A}$, aunque tienden a sentirse seguros y satisfechos de su elección, no saben si se mantendrán en ella. Es decir, contar con mayores recursos les permite mantenerse abiertos a la posibilidad de encontrar una opción que vaya más de acuerdo con ellos. Por lo tanto, si eligieron la correcta se encuentran satisfechos, en tanto que si aún no han descubierto la mejor opción se apoyan en sus recursos para proponerse un cambio.

Quienes no consideraron otras opciones y expresaron satisfacción, seguridad y la intención de mantenerse en la misma carrera, son particularmente los de identidad cerrada y los de rendimiento bajo. Así como los que ingresaron a la universidad B y a carreras de administración. Vemos que, estos adolescentes, una vez hecha su elección, tienden a mantenerse en ella sin cuestionarse.

Finalmente, solo los de lograda señalan sentirse muy satisfechos con su elección; asimismo, la mayoría de moratoria también expresa satisfacción con la decisión tomada, luego de contrastar sus opciones en los Estudios Generales. Al respecto, vemos que cuando el adolescente logra establecer un acuerdo entre las oportunidades que el entorno le ofrece (para que las compare y seleccione) y sus propias habilidades e intereses, alcanza un compromiso firme pero reflexivo, encontrando un nuevo equilibrio que le permite cumplir con la tarea vocacional, sintiéndose satisfecho con este logro.

Esta satisfacción es importante ya que no se trata de elegir una carrera solo por el tiempo que dure la universidad, sino que se elige a través de ella todo un proyecto de vida futuro, pues es con el rol ocupacional que el individuo participa en la sociedad y en el mundo adulto. La persona que no se siente satisfecha con lo que está haciendo difícilmente puede tener una calidad de vida profesional adecuada. Nos interesa, en tal sentido, poner el énfasis en el éxito no solo del quehacer, sino sobre todo del ser profesional.

Para ello es importante volver la atención hacia lo interno, hacia el conocimiento del sí mismo, descubriendo las habilidades e intereses personales, para luego buscar en el mundo externo las posibilidades que más se aproximen a ellos. En tal sentido, el egreso del colegio es un momento particularmente importante ya que da inicio a la vida "independiente" de los 
adolescentes, en el ámbito intelectual y afectivo, estableciendo varios patrones de madurez para su desenvolvimiento como personas adultas. Consideramos importante una investigación de este tema (el proceso seguido por el joven en el encuentro con su vocación) durante el tiempo de formación profesional en la universidad, particularmente durante el periodo de Estudios Generales, previo al ingreso a las respectivas facultades elegidas por los jóvenes.

Del mismo modo, consideramos que los datos aportados por este estudio abren nuevos espacios para facilitar un proceso saludable en el manejo de los estresores académicos y en la formación de la identidad vocacional. Al respecto, la implementación de estrategias para apoyar una transición adecuada y responsable en los jóvenes debe ir aunada con el de proporcionar un medio que no presione con intereses externos y ajenos al adolescente, sino que le permita redefinir su tarea en este periodo devolviendo el énfasis puesto hoy en día en el ingreso al encuentro de la vocación.

\section{REFERENCIAS}

Berzonsky, M. (1992). Identity style and coping strategies. Journal of Personality, 60 (4), 771-788.

Biehler, R. \& Snowman, J. (1992). Psicología aplicada a la enseñanza. México, D. F.: Limusa.
Blustein, D. \& Phillips, S. (1990). Relation between ego identity statuses and decision making styles. Journal of Counseling Psychology, 37 (2), 160-168.

Bohoslavsky, R. (1984). Orientación vocacional. La estrategia clínica. Buenos Aires: Nueva Visión.

Bosma, H. (1992). Identity in adolescence: Managing commitments. En G. Adams, T. Gullotta \& R. Montemayor (Eds.). Adolescent identity formation (pp. 91-121). Newbury Park, CA: Sage.

Carvajal, G. (1993). Adolecer: La aventura de una metamorfosis. Una visión psicoanalítica de la adolescencia. Bogotá: Tiresias.

Casullo, M.; Cayssials, A.; Liporace, M.; De Diuk, L.; Arce, J. \& Álvarez, L. (1996). Proyecto de vida y decisión vocacional. Buenos Aires: Paidós.

Erikson, E. (1993). El ciclo vital completado (3. ${ }^{\mathrm{a}}$ ed.). México, D.F.: Paidós.

Flum, H. (1994). Styles of identity formation in early and middle adolescence. Genetic, Social and General Psychology Monographs, 120 (4), 435-467.

Grotevant, H. \& Cooper, C. (1981). Assesing adolescent identity in the areas of occupation, religion, politics, friendship, dating and sex 
roles: Manual for administration and coding of the interview. JSAS Catalog of Selected Documents in Psychology, 11 (52).

Halleh, C. (1995). Rites of pasaje in adolescence. Adolescence,30 (120), 891-897.

Heymans, P. (1994). Developmental tasks: A cultural analysis of human development. En J. Laak, P. Heymans \& A. Podol'skij (Eds.). Developmental tasks: Towards a cultural analysis of human development. Dordrecht, Boston: Kluwer Academic Publishers.

Horrocks, J. (1996). Psicología de la adolescencia (5. ${ }^{\mathrm{a}}$ ed.). México, D.F.: Trillas.

Jara, L. (2003). "Estadios de la identidad vocacional y estrategias de afrontamiento en un grupo de estudiantes preuniversitarios". Tesis para optar el grado de licenciatura en Psicología. Lima: Pontificia Universidad Católica del Perú.

Jiménez, M. \& Maguiña, D. (1992). Las posibilidades de un espacio de reflexión psicoanalítica en un centro de estudios preuniversitarios. En M. Gheiler (Comp.). Psicoanálisis y educación (pp. 311-318). Lima: Biblioteca Peruana de Psicoanálisis.

Jones, R., Akers, J. \& White, J. (1994). Revised classification criteria for the Extended Objective Measure of
Ego Identity Status (EOMEIS). Journal of Adolescence, 17 (6), 533-549.

McLean, A. \& Flanigan, B. (1993). Transition-marking behaviors of adolescent males at first intercourse. Adolescence, 28 (111), 579-595.

Marcia, J. (1966). Development and validation of ego identity status. Journal of Personality and Social Psychology, 3 (5), 551-558.

Marcia, J. (1980). Identity in adolescence. En J. Adelson (Ed.). Handbook of Adolescent Psychology. Nueva York: Wiley.

Maurial, A. (1991). "Las posiciones de la identidad del Yo en un grupo de adolescentes tardíos de sector popular". Memoria para optar el grado de bachiller en Humanidades con mención en Psicología. Lima: Pontificia Universidad Católica del Perú.

Meeus, W. (1992). Toward a psychosocial analysis of adolescent identity: An evaluation of the epigenetic theory (Erikson) and the identity status model (Marcial). En W. Meeus, M. Goede, W. Kox \& K. Hurrelmann (Eds.). Adolescence, careers, and cultures. Berlín-Nueva York: Walter de Gruyter.

Meeus, W. (1996). Studies on identity development in adolescence: An overview of research and some new 
data. Journal of Youth and Adolescence, 25 (5), 569-598.

Osipow, S. (1991). Teorías sobre la elección de carreras. México, D.F.: Trillas.

Patterson, S.; Sochting, I. \& Marcia, J. (1992). The inner space and beyond: Women and identity. En G. Adams, T. Gullotta \& R. Montemayor (Eds.). Adolescent identity formation (pp. 9-24). Newbury Park, CA: Sage.
Stier, E. \& Clark, R. (1991). Adolescence: A literary pasaje. Adolescence, 26 (104), 757-768.

Trahtemberg, L. (1996). Educación para el tercer milenio. Lima: Bruño.

Waterman, A. (1993). Developmental perspectives on identity formation: from adolescence to adulthood. En J. Marcia, A. Waterman, D. Matteson, S. Archer \& J. Orlofsky (Eds.). Ego identity. Nueva York: Springer Verlag. 